\title{
Effects of Taekwondo Training on Body Composition: A Systematic Review and Meta-Analysis
}

\author{
Seunghui Baek ${ }^{1} \mathbb{D}$, Jong-Beom Park ${ }^{2}$, Sang-Hwan Choi ${ }^{2}$, Jae-Don Lee ${ }^{2}$ and Sang-Seok Nam ${ }^{2, *} \mathbb{D}$ \\ 1 Department of Health Exercise Management, Sungshin Women's University, Seoul 02844, Korea; \\ sh100@sungshin.ac.kr \\ 2 Taekwondo Research Institute of Kukkiwon, 32 Teheran 7-gil, Seoul 06130, Korea; \\ jb1907@hanmail.net (J.-B.P.); cshtkd@hanmail.net (S.-H.C.); drlee@kukkiwon.or.kr (J.-D.L.) \\ * Correspondence: playdata.n@gmail.com
}

check for

updates

Citation: Baek, S.; Park, J.-B.; Choi, S.-H.; Lee, J.-D.; Nam, S.-S. Effects of Taekwondo Training on Body

Composition: A Systematic Review and Meta-Analysis. Int. J. Environ. Res. Public Health 2021, 18, 11550. https://doi.org/10.3390/ ijerph182111550

Academic Editors: Omorogieva Ojo and Paul B. Tchounwou

Received: 31 August 2021

Accepted: 30 October 2021

Published: 3 November 2021

Publisher's Note: MDPI stays neutral with regard to jurisdictional claims in published maps and institutional affiliations.

Copyright: (C) 2021 by the authors Licensee MDPI, Basel, Switzerland. This article is an open access article distributed under the terms and conditions of the Creative Commons Attribution (CC BY) license (https:// creativecommons.org/licenses/by/ $4.0 /)$.

\begin{abstract}
Background: The purpose of this study is to investigate the effect of Taekwondo training on body composition and to evaluate the magnitude of the effect. Methods: Databases were used to select studies related to the effectiveness of Taekwondo training, and the inclusion criteria were as follows. Results: Thirty-seven studies were selected. We found statistically significant differences from the control group in weight, body mass index (BMI), waist circumference (WC), waist-hip ratio (WHR), body fat mass, body fat percentage, lean mass, and muscle mass. Also, the age group was statistically significant in control variables on weight, BMI, and body fat percentage. Conclusions: Taekwondo training had a positive effect on body composition, and these results suggest that Taekwondo training is an effective exercise method to lower obesity.
\end{abstract}

Keywords: Taekwondo; meta-analysis; body composition; obesity

\section{Introduction}

Insufficient physical activity was reported as the fourth most important risk factor among the causes of death worldwide [1]. For this reason, Korea has set physical activity goals for all age groups through the 3rd National Health Promotion Plan (HP2020) and is creating various policies and environments to promote physical activity [2]. According to the Korea Centers for Disease Control and Prevention report in 2019, the rate of aerobic exercise among adolescents increased by $3.0 \%$ from $10.9 \%$ in 2009 to $13.9 \%$ in 2018 . On the other hand, the rate of aerobic exercise among adults over the age of 19 and older decreased by $9.8 \%$ from $58.3 \%$ in 2014 to $48.5 \%$ in 2017 , and the rate of walking among adults over the age of 19 and older decreased by $6.7 \%$ from $45.7 \%$ in 2007 to $39.0 \%$ in 2017 [3]. However, according to the study of Guthold et al. [4], a 2016 survey of 298 schools in 146 countries found that $81.0 \%$ of $11-17$ year olds were not getting enough physical activity. On the other hand, in the case of Korea, $94.2 \%$ of adolescents showed insufficient amount of physical activity, which made Korea recognized as the country with the most insufficient amount of physical activity for adolescents in the world. Since such a decrease in physical activity can cause a decrease in physical strength and muscle mass [5] and an increase in body fat mass, thereby causing metabolic diseases [6], efforts to increase physical activity are required.

Taekwondo is a traditional martial art from Korea and is recognized as a global martial arts and sport with more than 100 million practitioners in 210 countries worldwide [1] Taekwondo as an exercise has positive effects on the psychological and physiological areas for the growth and development of children and adolescents. In addition, Taekwondo training prevents or positively improves obesity, dyslipidemia, diabetes, hypertension, cerebrovascular, and cardiovascular diseases in adults and the elderly [7-10]. In addition, it is expected to improve various physical strengths, including aerobic capacity, muscle strength, muscular endurance, flexibility, speed, and agility through the physiological effects of Taekwondo practice [11-14]. For this reason, Taekwondo is considered suitable 
as an essential exercise for improving the physical activity of Koreans and preventing and improving various diseases. However, although multiple studies have been conducted to verify the effectiveness of Taekwondo training, the results are not consistent, and depend on the study subject, method and duration of interventions, making it difficult to generalize the results. Therefore, it is necessary to present the best evidence to prove the effect of physical activity through Taekwondo among Koreans [15]. Thus, this study shows the effects of Taekwondo training on changes in body composition comprehensively and quantitatively to serve as evidence for the development of Taekwondo training as a war to improve obesity caused by lack of exercise in modern people including not only Koreans but people worldwide.

\section{Materials and Methods}

This study was conducted with reference to the Preferred Reporting for Systematic Reviews and Meta-Analysis (PRISMA) guidelines [16].

\subsection{Search Strategy}

In this study, we searched the literature in electronic databases, including the Cochrane Library, EMBASE, and PubMed, and used RISS (Research Information Sharing Service), NDSL (National Science and Technology Information Center) in Korea. The keyword used in the literature search was "Taekwondo". In addition, we included gray literature such as dissertations to reduce publication bias. The language of the papers was limited to Korean and English. The publication year was not limited to the default setting. The reason we used only 'taekwondo' as a keyword is to prevent the omission of literature related to this topic as much as possible.

\subsection{Selection of Studies}

We selected literature according to the following PICO (participants, interventions, comparisons, outcomes) criteria. We included studies where the study subjects were the general public and excluded studies where the study subjects were not the general public (athletes, disabled people, hypertensive patients, diabetic patients, etc.). In addition, we selected studies where the intervention method was Taekwondo training and excluded studies that added interventions other than Taekwondo. Also, we included only studies with a control group who did not practice Taekwondo. We selected studies that presented actual measurements of body composition and studies with an RCT (randomized controlled trial) study design.

\subsection{Moderator}

The effects of Taekwondo intervention were analyzed by looking at how the results were influenced by the differences in the moderators, including gender, age, training period, research method, types of literature, and quality evaluation. Age was divided into three groups: students who attend elementary, middle, and high school, adults, and the elderly. The training periods were divided into 12 weeks or less and more than 12 weeks. Publication types were identified by dividing them into degree thesis and journal publications.

\subsection{Quality Assessment}

For the qualitative evaluation of the selected literature, the Cochrane Collaboration's RoB (Risk of Bias) tool [16] was used. The evaluation consists of 7 items, including: (1) generating order of random assignment; (2) hiding assignment order; (3) blinding study participants and researchers; (4) blinding outcome evaluation; (5) insufficient result data; (6) selective reporting; and (7) other biases. The evaluation was rated as low risk, high risk, and uncertain. 


\subsection{Statistical Analysis}

The effect size of the Taekwondo intervention and the heterogeneity were analyzed using the R program (version 3.6.2, R Foundation for Statistical Computing, Vienna, Austria) [17]. The outcome variables were all continuous variables and included Height, weight, BMI, WC, WHR, fat mass, lean mass, body fat percentage, and muscle mass. The overall effect size was analyzed when there were three or more outcome variables. The measurement unit was the same, expressed as mean difference (MD) and $95 \%$ confidence intervals (Cls). The random effects model was used under the assumption that the outcome variables of each study were heterogeneous [18]. Meta-ANOVA and meta-regression were performed for the moderating variables, and both continuous variables (sample sizes) and categorical variables (gender, age, study period, publication type) were estimated using the Restricted Maximum Likelihood (REML) method [19].

\subsection{Assessment of Heterogeneity}

Heterogeneity was assessed using the Cochrane $Q$ test and Higgins' $\mathrm{I}^{2}$ statistics. When the $p$-value of the Cochrane $\mathrm{Q}$ test was 0.1 or less or the Higgins' $\mathrm{I}^{2}$ was $50 \%$ or more, it was considered that there was statistically significant heterogeneity.

\subsection{Publication Bias}

For the publication bias analysis, we applied a funnel plot using standard error and MD, additionally applied Egger's test and Begg's test [20].

\section{Result}

\subsection{Inclusion of Studies}

Of the 803 studies, we selected 171 studies, excluding duplicate studies (280) and those unrelated to this study (352). Then, as a result of full-text screening according to the PICO criteria, 131 studies without a control group and three studies that did not explain the Taekwondo training program were excluded. Therefore, we finally selected 37 studies for meta-analysis. All 37 papers were satisfied for both qualitative and quantitative analysis. The detailed process is shown in Figure 1.

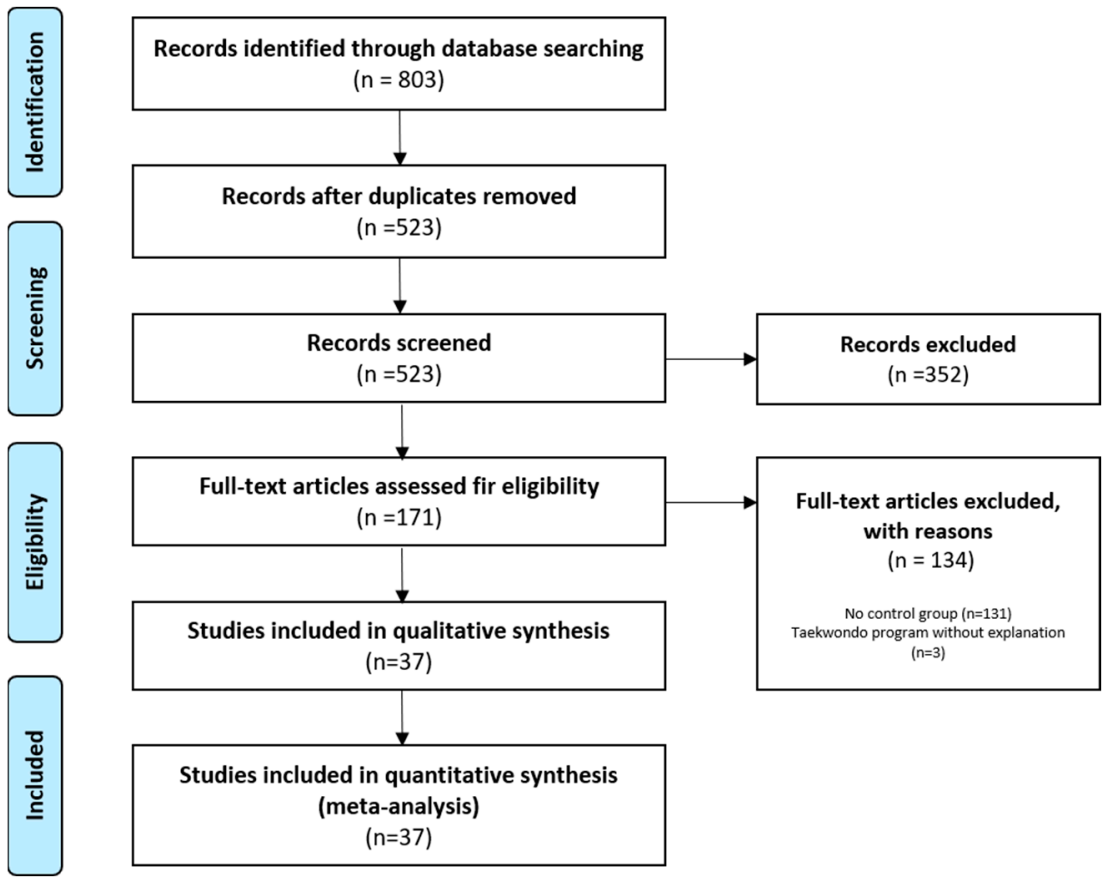

Figure 1. PRISMA flow diagram. 


\subsection{Characteristics of the Included Studies}

The total sample size was 801 . The average age of the participants was 32.19 years old, ranging from 7.46 to 75.07 , and $54.2 \%$ of the participants were male. 18 studies [21-37] were for students, 12 studies [38-49] were for adults, and 7 studies were for seniors [50-56], and the training period was 8-24 months. According to the publication type, 15 were for dissertations studies, and 22 studies were for journal publication. The characteristics of the included studies are summarized in Table 1.

\subsection{Quality Assessment}

Two independent researchers (S.B., S.N.) performed quality assessment as shown in Figure 2, based on the criteria of the Cochrane Collaboration. All 37 studies were randomized control studies, and all studies analyzed the results, excluding participants who dropped out during the intervention.

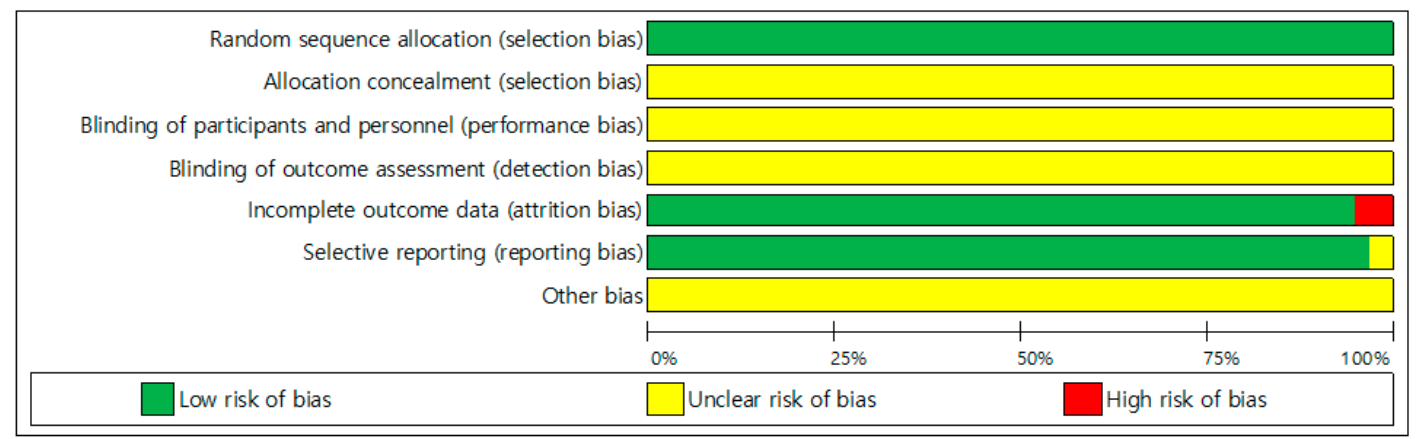

Figure 2. Assessment of risk of bias in included studies.

\subsection{Outcome Findings}

\subsubsection{Height}

Sixteen studies reported height (total $n=184$; taekwondo training group $n=168$, control group $n=166$ ). The mean difference (MD) in heights between the Taekwondo training and the control group was $0.274(95 \% \mathrm{CI} ;-0.974,1.521)$, and no statistically significant difference was found. For the heterogeneity test, the results of Cochrane $\mathrm{Q}$ were $p=1.00$, and Higgins' $\mathrm{I}^{2}$ was $0 \%$ (Figure 3a).

\begin{tabular}{|c|c|c|c|c|}
\hline Study & Taekwondo & Control & MD & $95 \% \mathrm{Cl}$ \\
\hline Choi W, 2000 & 9 & 9 & 0.100 & {$[-6.766 ; 6.966]$} \\
\hline An SW, 2004 & 10 & 10 & 1.020 & {$[-4.585 ; 6.625]$} \\
\hline Lee SH, 2008 & 8 & 8 & 0.500 & {$[-5.198 ; 6.198]$} \\
\hline Moon DS, 2008 & 9 & 9 & 0.850 & {$[-5.210 ; 6.910]$} \\
\hline Lee SW, 2011 & 8 & 8 & -0.460 & {$[-9.661 ; 8.741]$} \\
\hline Jung SH, 2012 & 10 & 10 & -0.700 & {$[-6.395 ; 4.995]$} \\
\hline Jung HC, 2014 & 13 & 12 & -0.100 & {$[-6.160 ; 5.960]$} \\
\hline Kang HJ, 2014 & 11 & 13 & 0.100 & {$[-3.314 ; 3.514]$} \\
\hline Lee SH, 2015 & 9 & 9 & -0.230 & {$[-6.330 ; 5.870]$} \\
\hline Moon DS, 2007 & 10 & 12 & 0.400 & {$[-2.355 ; 3.155]$} \\
\hline Kim WK, 2009 & 10 & 10 & 0.580 & {$[-4.791 ; 5.951]$} \\
\hline Kwon YC, 2010 & 12 & 12 & 0.970 & {$[-5.040 ; 6.980]$} \\
\hline Moon DS, 2010 & 15 & 15 & 0.260 & {$[-4.063 ; 4.583]$} \\
\hline Shin JD, 2010 & 7 & 7 & 0.840 & {$[-7.050 ; 8.730]$} \\
\hline Song JK, 2013 & 12 & 7 & -0.100 & {$[-6.047 ; 5.847]$} \\
\hline Joo SE, 2017 & 15 & 15 & 0.100 & {$[-4.105 ; 4.305]$} \\
\hline Total (fixed effect, 95\% Cl) & & & 0.274 & {$[-0.974 ; 1.521]$} \\
\hline Total (random effects, 95\% Cl) & & & 0.274 & {$[-0.974 ; 1.521]$} \\
\hline
\end{tabular}

(a)

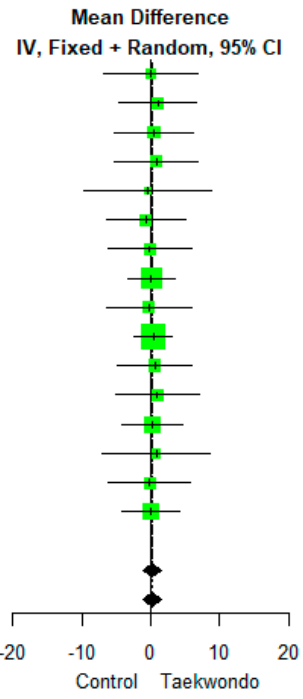

Figure 3. Cont. 
Study

Choi W, 2000

An SW, 2004

Lee SH, 2008

Moon DS, 2008

Chea SI, 2011

Lee SW, 2011

Jung SH, 2012

Jung HC, 2014

Kang HJ, 2014

Kang MG, 2014

Lee SJ, 2014

Lee SH, 2015

Chea SI, 2016

Seo DK, 2016

Seo DW, 2019

Moon DS, 2007

Kim WK, 2009

Kim KT, 2010

Kwon YC, 2010

Moon DS, 2010

Shin JD, 2010

Lee KK, 2011

Lee SH, 2011

Cho WJ, 2012

Cho WJ, 2013

Song JK, 2013

Cho WJ, 2014

Lee KK, 2015

Joo SE, 2017

Lee KS, 2017

Lim YR, 2017

Jung MK, 2023

Kim NS(a), 2018

Kim NS(b), 2018

$\begin{array}{ccrr}\text { Taekwondo } & \text { Control } & \text { MD } & 95 \% \mathrm{Cl} \\ 9 & 9 & 2.800 & {[-5.076 ; 10.676]} \\ 10 & 10 & -0.710 & {[-7.724 ; 6.304]} \\ 8 & 8 & -1.290 & {[-8.591 ; 6.011]} \\ 9 & 9 & -2.790 & {[-10.930 ; 5.350]} \\ 8 & 8 & -3.980 & {[-8.991 ; 1.031]} \\ 8 & 8 & -2.400 & {[-9.976 ; 5.176]} \\ 10 & 10 & -3.600 & {[-8.125 ; 0.925]} \\ 13 & 12 & -4.500 & {[-13.617 ; 4.617]} \\ 11 & 13 & 0.000 & {[-4.032 ; 4.032]} \\ 10 & 10 & -5.470 & {[-7.103 ;-3.837]} \\ 10 & 10 & -3.590 & {[-8.133 ; 0.953]} \\ 9 & 9 & -1.900 & {[-8.305 ; 4.505]} \\ 8 & 8 & -0.650 & {[-9.638 ; 8.338]} \\ 13 & 13 & -1.670 & {[-7.150 ; 3.810]} \\ 23 & 22 & -3.840 & {[-7.298 ;-0.382]} \\ 10 & 12 & -0.400 & {[-2.999 ; 2.199]} \\ 10 & 10 & -5.310 & {[-8.965 ;-1.655]} \\ 10 & 10 & -3.650 & {[-8.373 ; 1.073]} \\ 12 & 12 & -2.370 & {[-6.954 ; 2.214]} \\ 15 & 15 & -2.210 & {[-7.771 ; 3.351]} \\ 7 & 7 & 1.260 & {[-5.928 ; 8.448]} \\ 12 & 12 & -0.010 & {[-4.369 ; 4.349]} \\ 6 & 9 & -1.930 & {[-11.969 ; 8.109]} \\ 13 & 13 & -1.730 & {[-3.719 ; 0.259]} \\ 12 & 12 & -3.820 & {[-5.325 ;-2.315]} \\ 12 & 7 & 0.200 & {[-10.495 ; 10.895]} \\ 10 & 10 & -4.080 & {[-5.788 ;-2.372]} \\ 20 & 20 & -1.170 & {[-5.446 ; 3.106]} \\ 15 & 15 & -1.200 & {[-6.198 ; 3.798]} \\ 8 & 7 & -0.550 & {[-10.345 ; 9.245]} \\ 15 & 15 & -0.980 & {[-7.298 ; 5.338]} \\ 8 & 9 & -1.060 & {[-6.284 ; 4.164]} \\ 6 & 10 & -1.700 & {[-19.790 ; 16.390]} \\ 5 & 9 & -2.700 & {[-18.765 ; 13.365]}\end{array}$

Total (fixed effect, 95\% CI)

Total (random effects, $95 \% \mathrm{CI}$ )

Heterogeneity: $\mathrm{Tau}^{2}=0 ; \mathrm{Chi}^{2}=30.56, \mathrm{df}=33(\mathrm{P}=0.59) ; \mathrm{I}^{2}=0 \%$

$-3.085 \quad[-3.720 ;-2.449]$

$-3.085[-3.720 ;-2.449]$

(b)

Study

Choi W, 2000

Moon DS, 2008

Chea SI, 2011

Lee SW, 2011

Jung SH, 2012

Jung HC, 2014

Kang MG, 2014

Lee SJ, 2014

Lee SH, 2015

Seo DK, 2016

Han SY, 2007

Moon DS, 2007

Kim WK, 2009

Shin JD, 2009

Kwon YC, 2010

Moon DS, 2010

Cho WJ, 2013

Lee KS, 2017

Lee KS, 2017

Total (fixed effect, 95\% Cl)

Total (random effects, 95\% Cl)

Heterogeneity: $\mathrm{Tau}^{2}=0 ; \mathrm{Chi}^{2}=12.47, \mathrm{df}=18(\mathrm{P}=0.82) ; \mathrm{I}^{2}=0 \%$

Taekwondo Control MD $\quad 95 \% \mathrm{Cl}$

$9 \quad 9 \quad-1.500[-2.804 ;-0.196]$

$\begin{array}{rrrr}9 & 9 & -1.620[-4.441 ; 1.201] \\ 8 & 8 & 0.120[-1.806 ; 2.046]\end{array}$

$8 \quad 8 \quad-0.770[-1.982 ; 0.442]$

$10 \quad 10 \quad-2.200[-4.787 ; 0.387]$

$13 \quad 12 \quad-1.500[-3.279 ; 0.279]$

$10 \quad 10-1.990[-3.208 ;-0.772]$

$10 \quad 10 \quad-2.030[-3.227 ;-0.833]$

$9 \quad 9 \quad-0.900[-4.153 ; 2.353]$

$1313-0.600[-2.499 ; 1.299]$

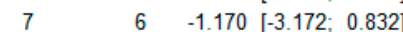

$10 \quad 12 \quad-2.200[-4.414 ; 0.014]$

$10 \quad 10 \quad-2.400[-3.850 ;-0.950]$

$10 \quad 10 \quad 0.300[-2.054 ; 2.654]$

$12 \quad 12-1.430[-2.582 ;-0.278]$

$1515-1.010[-2989 ; 0.969]$

$1212-1.650[-2.107 ;-1.193]$

$15 \quad 15 \quad-1.050[-3.287 ; 1.187]$

$8 \quad 7 \quad-0.380[-3.055 ; 2.295]$

$-1.503[-1.803 ;-1.204]$

$1.503[-1.803 ;-1.204]$

(c)
Mean Difference

IV, Fixed + Random, $95 \% \mathrm{Cl}$

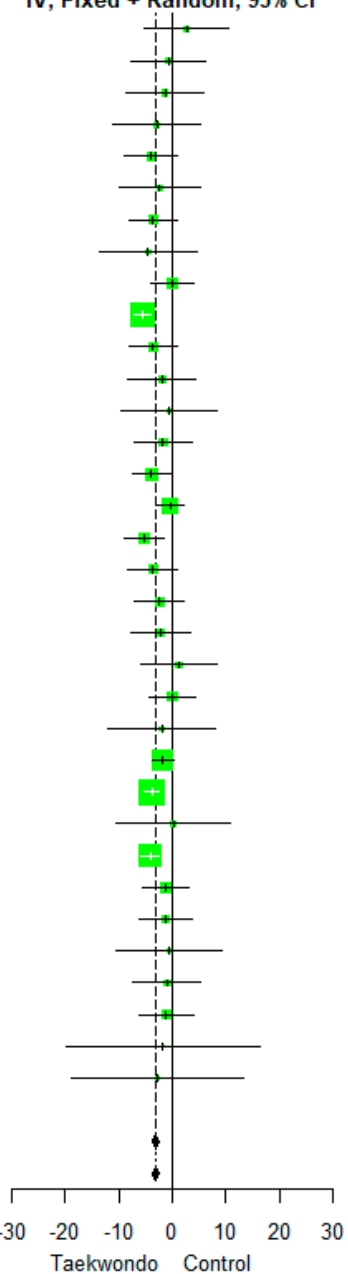

Mean Difference

IV, Fixed + Random, 95\% Cl

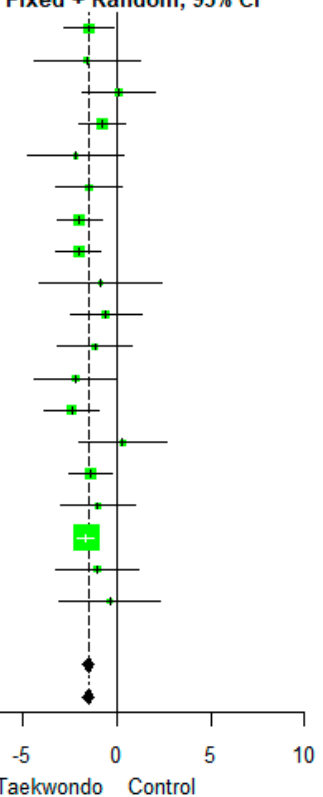

Figure 3. Cont. 


\begin{tabular}{|c|c|c|c|c|}
\hline Study & Taekwondo & Control & MD & $95 \%$ \\
\hline Lee SW, 2011 & 8 & 8 & -1.940 & {$[-6.120 ; 2.2$} \\
\hline Jung HC, 2014 & 13 & 12 & -5.000 & {$[-11.719 ; 1.7$} \\
\hline Kwon YC, 2010 & 12 & 12 & -3.830 & {$[-7.309 ;-0.3$} \\
\hline Lee KK, 2015 & 20 & 20 & -2.300 & {$[-6.521 ; 1.9$} \\
\hline Jung MK, 2022 & 8 & 9 & -1.100 & {$[-3.651 ; 1.4$} \\
\hline Kim NS(b), 2018 & 5 & 9 & -1.300 & {$[-12.841 ; 10.2$} \\
\hline Total (fixed effect, 95\% CI) & & & -2.231 & {$[-3.855 ;-0.6$} \\
\hline Total (random effects, 95\% Cl) & & & -2.231 & {$[-3.855 ;-0$.} \\
\hline Heterogeneity: $\mathrm{Tau}^{2}=0 ; \mathrm{Chi}^{2}=2.26$ & $6, d f=5(P=0.8$ & $=0 \%$ & & \\
\hline
\end{tabular}

(d)

Study

An SW, 2004

Han SY, 2007

Moon DS, 2007

Kim WK, 2009

Kwon YC, 2010

Lee KK, 2015

Joo SE, 2017

Lee KS, 2017

Jung MK, 2024

Total (fixed effect, 95\% Cl)

Total (random effects, 95\% $\mathrm{CI}$ )

Heterogeneity: $\mathrm{Tau}^{2}=0.0006 ; \mathrm{Chi}^{2}=24.48, \mathrm{df}=9(\mathrm{P}<0.01) ; \mathrm{I}^{2}=63 \%$

Taekwondo Control MD $95 \%$

10

7

10

10

12

20

15

8

8

5

19]

$10-0.060[-0.104 ;-0.016]$ $-0.020[-0.058 ; 0.018]$ $-0.100[-0.135 ;-0.065]$ $0.000[-0.049 ; 0.049]$ $-0.020[-0.046 ; 0.006]$ $-0.030[-0.074 ; 0.014]$ $-0.030[-0.066 ; 0.006]$ $0.000[-0.029 ; 0.029]$ $-0.010[-0.065 ; 0.045]$

$-0.028[-0.039 ;-0.017]$ $-0.029[-0.048 ;-0.010]$

Mean Difference

IV, Fixed + Random, 95\% Cl

51]

\section{1]}

\section{(n)}

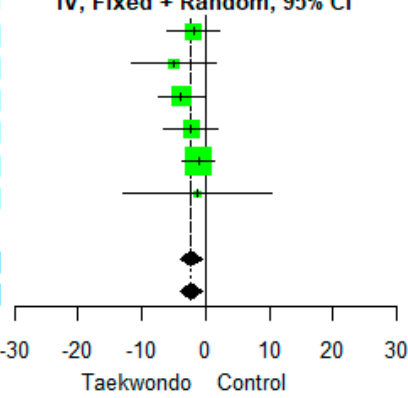

Mean Difference

IV, Fixed + Random, $95 \% \mathrm{CI}$

Kim NS(b), 2018 $-0.020[-0.049 ; 0.009]$
Study

Moon DS, 2008

Jung SH, 2012

Jung HC, 2014

Kang HJ, 2014

Seo DW, 2019

Shin JD, 2009

Kwon YC, 2010

Lee KK, 2011

Song JK, 2013

Joo SE, 2017

Lee KS, 2017

Lim YR, 2017

Jung MK, 2019

Kim NS(a), 2018 (e)

\begin{tabular}{|c|c|c|c|}
\hline Taekwondo & Control & MD & $95 \% \mathrm{Cl}$ \\
\hline 9 & 9 & -2.380 & {$[-6.356 ; 1.596]$} \\
\hline 10 & 10 & -0.700 & {$[-2.490 ; 1.090]$} \\
\hline 13 & 12 & -2.400 & {$[-6.265 ; 1.465]$} \\
\hline 11 & 13 & -0.300 & {$[-2.779 ; 2.179]$} \\
\hline 23 & 22 & -2.260 & {$[-7.190 ; 2.670]$} \\
\hline 10 & 10 & -2.200 & {$[-5.703 ; 1.303]$} \\
\hline 12 & 12 & -3.810 & {$[-5.470 ;-2.150]$} \\
\hline 12 & 12 & -0.790 & {$[-4.006 ; 2.426]$} \\
\hline 12 & 7 & 0.300 & {$[-5.523 ; 6.123]$} \\
\hline 15 & 15 & -1.300 & {$[-4.120 ; 1.520]$} \\
\hline 8 & 7 & -1.310 & {$[-8.526 ; 5.906]$} \\
\hline 15 & 15 & -0.470 & {$[-4.658 ; 3.718]$} \\
\hline 8 & 9 & -0.430 & {$[-1.784 ; 0.924]$} \\
\hline \multirow[t]{3}{*}{6} & 10 & -1.100 & {$[-9.095 ; 6.895]$} \\
\hline & & -1.398 & {$[-2.109 ;-0.687]$} \\
\hline & & -1.398 & {$[-2.109 ;-0.687]$} \\
\hline
\end{tabular}

(f)

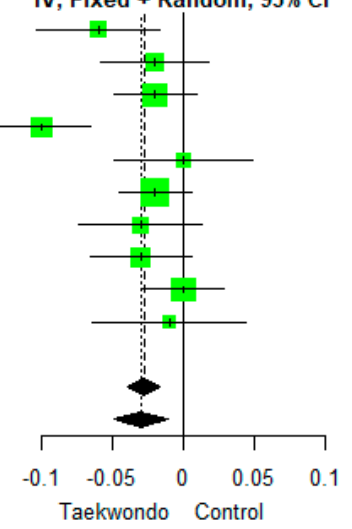

Mean Difference

IV, Fixed + Random, $95 \% \mathrm{Cl}$

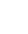

Total (fixed effect, 95\% $\mathrm{Cl}$ )

Total (random effects, $95 \% \mathrm{Cl}$ )

Heterogeneity: $\mathrm{Tau}^{2}=0 ; \mathrm{Chi}^{2}=12.89, \mathrm{df}=13(\mathrm{P}=0.46) ; I^{2}=0 \%$

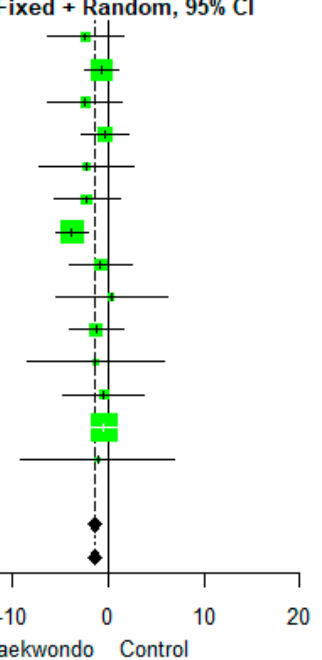

Figure 3. Cont. 
Study

Choi W, 2000

An SW, 2004

Lee SH, 2008

Moon DS, 2008

Chea SI, 2011

Lee SW, 2011

Jung SH, 2012

Jung HC, 2014

Kang HJ, 2014

Kang MG, 2014

Lee SJ, 2014

Lee SH, 2015

Chea SI, 2016

Seo DK, 2016

Han SY, 2007

Moon DS, 2007

Kim WK, 2009

Shin JD, 2009

Kim KT, 2010

Kwon YC, 2010

Moon DS, 2010

Shin JD, 2010

Lee KK, 2011

Lee SH, 2011

Cho WJ, 2012

Cho WJ, 2013

Song JK, 2013

Cho WJ, 2014

Lee KK, 2015

Joo SE, 2017

Lee KS, 2017

Lim YR, 2017

Jung MK, 2018

Kim NS(a), 2018

Kim NS(b), 2018

Lee JK, 2019
Taekwondo Control MD $\quad 95 \% \mathrm{Cl}$

$9 \quad 9 \quad-3.900[-9.450 ; 1.650]$

$10 \quad 10-0.580[-2.561 ; 1.401]$

$8 \quad 8 \quad-2.000[-9.552 ; 5.552]$

$9 \quad 9-3.350[-8.183 ; 1.483]$

$8 \quad 8 \quad-1.440[-6.240 ; 3.360]$

$8 \quad 8 \quad-5.530[-9.215 ;-1.845]$

$10 \quad 10 \quad-5.400[-9.564 ;-1.236]$

$13 \quad 12-1.600[-5.499 ; 2.299]$

$11 \quad 13-0.600[-3.509 ; 2.309]$

$10 \quad 10 \quad-3.270[-4.341 ;-2.199]$

$10 \quad 10 \quad-3.350[-4.589 ;-2.111]$

$9 \quad 9 \quad-4.250[-12.556 ; 4.056]$

$8 \quad 8 \quad-2.450[-7.213 ; 2.313]$

$13 \quad 13-0.790[-3.764 ; 2.184]$

$7 \quad 6 \quad-2.830[-6.148 ; 0.488]$

$10 \quad 12-1.900[-4.508 ; 0.708]$

$10 \quad 10 \quad-2.120[-5.567 ; 1.327]$

$10 \quad 10 \quad-3.900[-7.908 ; 0.108]$

$-3.230[-6.176 ;-0.284]$

$-6.150[-8.102 ;-4.198]$

$-1.310[-4.815 ; 2.195]$

$-1.210 \quad[-5.632 ; 3.212]$

$-0.200 \quad[-4.182 ; 3.782]$

$0.750 \quad[-4.681 ; 6.181]$

$-3.900[-4.825 ;-2.975]$

$-2.980[-3.765 ;-2.195]$

$1.000 \quad[-5.294 ; 7.294]$

$-2.840 \quad[-9.251 ; 3.571]$

$-2.560[-5.706 ; 0.586]$

$-1.900 \quad[-4.180 ; 0.380]$

$-0.910 \quad[-7.492 ; 5.672]$

$-2.040 \quad[-5.661 ; 1.581]$

$-3.060[-6.640 ; 0.520]$

$-0.400[-4.772 ; 3.972]$

$-0.600 \quad[-5.680 ; 4.480]$

$-3.300[-6.253 ;-0.347]$

$-2.996[-3.376 ;-2.615]$

-2.850 [ $-3.327 ;-2.373]$

Total (random effects, 95\% C

Heterogeneity: Tau $^{2}=0.2479 ; \mathrm{Chi}^{2}=40.82, \mathrm{df}=35(\mathrm{P}=0.23) ; I^{2}=14 \%$

(g)
Mean Difference

IV, Fixed + Random, $95 \% \mathrm{Cl}$

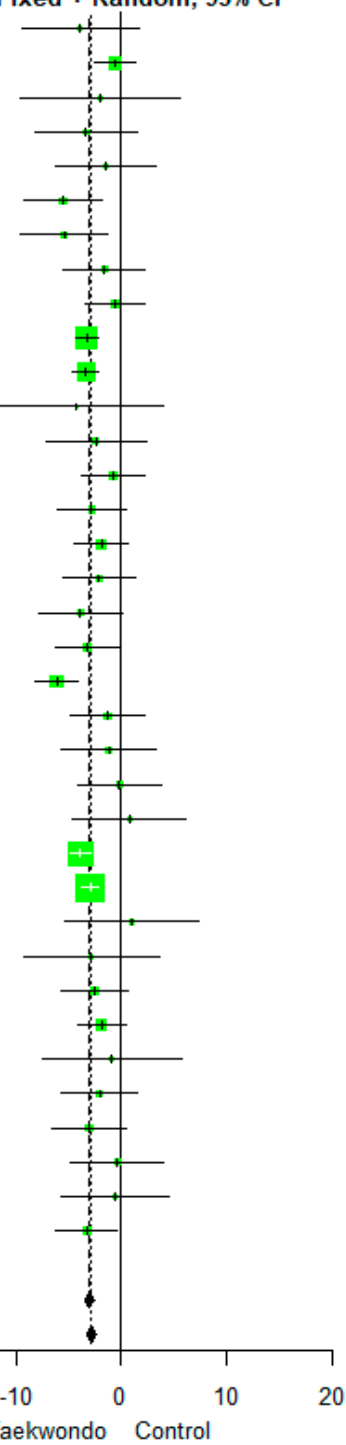

Taekwondo Control

Figure 3. Cont. 


\begin{tabular}{|c|c|c|c|c|}
\hline Study & Taekwondo & Control & MD & $95 \% \mathrm{Cl}$ \\
\hline An SW, 2004 & 10 & 10 & 0.930 & {$[-3.613 ; 5.473]$} \\
\hline Lee SH, 2008 & 8 & 8 & -0.010 & {$[-3.213 ; 3.193]$} \\
\hline Moon DS, 2008 & 9 & 9 & -0.400 & {$[-5.117 ; 4.317]$} \\
\hline Lee SW, 2011 & 8 & 8 & 1.410 & {$[-2.971 ; 5.791]$} \\
\hline Jung SH, 2012 & 10 & 10 & 0.700 & {$[-2.421 ; 3.821]$} \\
\hline Jung HC, 2014 & 13 & 12 & -4.300 & {$[-11.207 ; 2.607]$} \\
\hline Kang HJ, 2014 & 11 & 13 & 0.400 & {$[-1.893 ; 2.693]$} \\
\hline Lee SH, 2015 & 9 & 9 & 0.230 & {$[-2.875 ; 3.335]$} \\
\hline Seo DK, 2016 & 13 & 13 & -0.690 & {$[-3.411 ; 2.031]$} \\
\hline Seo DW, 2019 & 23 & 22 & 5.730 & [ $2.223 ; 9.237]$ \\
\hline Han SY, 2007 & 7 & 6 & -0.540 & {$[-2.770 ; 1.690]$} \\
\hline Moon DS, 2007 & 10 & 12 & 1.300 & {$[-0.961 ; 3.561]$} \\
\hline Kim WK, 2009 & 10 & 10 & 4.500 & [ $1.807 ; 7.193]$ \\
\hline Kim KT, 2010 & 10 & 10 & 3.200 & {$[1.814 ; 4.586]$} \\
\hline Kwon YC, 2010 & 12 & 12 & 1.430 & {$[-2.015 ; 4.875]$} \\
\hline Moon DS, 2010 & 15 & 15 & 2.550 & {$[-0.777 ; 5.877]$} \\
\hline Shin JD, 2010 & 7 & 7 & 2.320 & {$[-2.011 ; 6.651]$} \\
\hline Lee KK, 2011 & 12 & 12 & 0.750 & {$[-2.717 ; 4.217]$} \\
\hline Song JK, 2013 & 12 & 7 & -1.100 & {$[-7.119 ; 4.919]$} \\
\hline Lee KK, 2015 & 20 & 20 & 0.490 & {$[-1.016 ; 1.996]$} \\
\hline Joo SE, 2017 & 15 & 15 & 0.400 & {$[-2.067 ; 2.867]$} \\
\hline Jung MK, 2021 & 8 & 9 & 0.060 & {$[-2.608 ; 2.728]$} \\
\hline Kim NS(a), 2018 & 6 & 10 & -1.200 & {$[-10.999 ; 8.599]$} \\
\hline Kim NS(b), 2018 & 5 & 9 & -1.100 & {$[-9.339 ; 7.139]$} \\
\hline Total (fixed effect, $95 \% \mathrm{CI}$ ) & & & 1.234 & {$[0.661 ; 1.808]$} \\
\hline Total (random effects, $95 \% \mathrm{Cl}$ ) & & & 1.109 & {$[0.366 ; 1.851]$} \\
\hline \multicolumn{5}{|c|}{ Heterogeneity: $\mathrm{Tau}^{2}=0.9086 ; \mathrm{Chi}^{2}=32.77, \mathrm{df}=23(\mathrm{P}=0.09) ; \mathrm{I}^{2}=30 \%$} \\
\hline
\end{tabular}

(h)

Taekwondo Co
8
10
23
10
6
15
12

Mean Difference

IV, Fixed + Random, $95 \% \mathrm{Cl}$

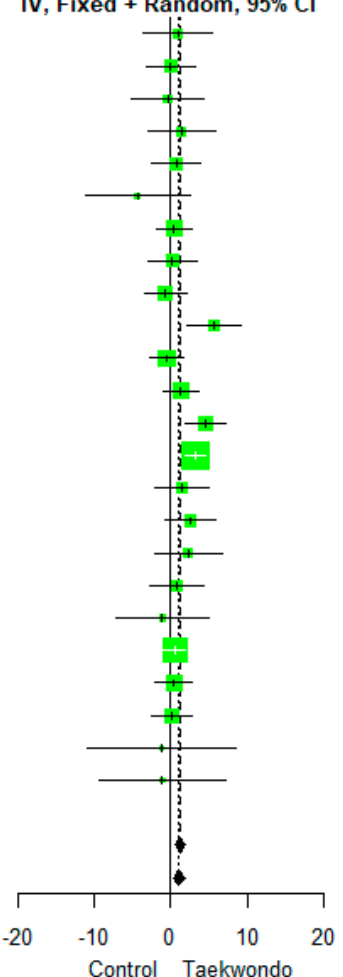

Mean Difference

IV, Fixed + Random, $95 \% \mathrm{CI}$

$8 \quad 0.400[-2.526 ; 3.326]$

$10 \quad 0.400[-2.429 ; 3.229]$

$4.690[1.920 ; 7.460]$

$2.500[0.594 ; 4.406]$

$-2.100[-8.013 ; 3.813]$

$-0.070[-2.164 ; 2.024]$

$4.100[2.130 ; 6.070]$

Total (fixed effect, $95 \% \mathrm{Cl}$ )

Total (random effects, 95\% Cl)

Heterogeneity: $\mathrm{Tau}^{2}=2.7895 ; \mathrm{Chi}^{2}=16.22, \mathrm{df}=6(\mathrm{P}=0.01) ; \mathrm{I}^{2}=63 \%$

$2.046[1.118 ; 2.974]$

$1.818[0.208 ; 3.427]$

(i)

Figure 3. Forest plot of the mean difference in physique and body composition. (a) Height; (b) weight; (c) body mass index; (d) waist circumference; (e) waist-hip ratio; (f) body fat mass; (g) body fat percentage; (h) lean mass; (i) muscle mass. 
Table 1. Characteristics of included studies.

\begin{tabular}{|c|c|c|c|c|c|c|c|c|c|c|}
\hline Study & Study Type & $\begin{array}{l}\text { Frequency } \\
\text { (Day/Week) }\end{array}$ & Participants & Sex & \multicolumn{2}{|c|}{ Taekwondo $(n$, Age) } & \multicolumn{2}{|c|}{ Control $(n$, Age) } & \multirow{2}{*}{$\begin{array}{c}\begin{array}{c}\text { Body } \\
\text { Composition } \\
\text { Method }\end{array} \\
\text { Skinfold } \\
\end{array}$} & \multirow{2}{*}{$\begin{array}{c}\text { Outcome Variable } \\
\% \text { Body fat, BMI, Height, Weight }\end{array}$} \\
\hline Choi W, 2000 [21] & Thesis & $3 / 24$ & $\begin{array}{l}\text { Elementary } \\
\text { student, } 40 \% \\
\text { body fat } \uparrow\end{array}$ & M & 9 & 11.78 & 9 & 11.89 & & \\
\hline Lee SH, 2008 [22] & Thesis & $5 / 12$ & $\begin{array}{l}\text { Elementary } \\
\text { student }\end{array}$ & M & 8 & 11.1 & 8 & 11.1 & BIA & $\begin{array}{l}\text { \% Body fat, Height, Lean mass, } \\
\text { Muscle mass, Weight }\end{array}$ \\
\hline Lee SW, 2011 [23] & Thesis & $5 / 12$ & $\begin{array}{c}\text { Elementary } \\
\text { student }\end{array}$ & Mixed & 8 & 11.13 & 8 & 10.88 & BIA & $\begin{array}{l}\text { \% Body fat, BMI, Height, Lean } \\
\text { mass, WC, Weight }\end{array}$ \\
\hline Jung SH, 2012 [24] & Thesis & $5 / 12$ & $\begin{array}{l}\text { Elementary } \\
\text { student }\end{array}$ & M & 10 & 10.2 & 10 & 10.3 & BIA & $\begin{array}{c}\text { \% Body fat, BMI, Fat mass, } \\
\text { Height, Lean mass, Muscle mass, } \\
\text { Weight }\end{array}$ \\
\hline Lee SJ, 2014 [25] & Thesis & $3 / 16$ & $\begin{array}{l}\text { Elementary } \\
\text { student, } 25 \% \\
\text { body fat } \uparrow\end{array}$ & M & 10 & 12.6 & 10 & 12.7 & BIA & $\%$ Body fat, BMI, Weight \\
\hline Lee SH, 2015 [26] & Thesis & $5 / 12$ & $\begin{array}{c}\text { Elementary } \\
\text { student }\end{array}$ & M & 9 & 7.46 & 9 & 7.74 & BIA & $\begin{array}{l}\text { \% Body fat, BMI, Height, Lean } \\
\text { mass, Weight }\end{array}$ \\
\hline Seo DW, 2019 [27] & Thesis & $5 / 12$ & $\begin{array}{c}\text { Elementary } \\
\text { student }\end{array}$ & M & 23 & 10 & 22 & 9.67 & BIA & $\begin{array}{c}\text { Fat mass, Lean mass, Muscle } \\
\text { mass, Weight }\end{array}$ \\
\hline An SW, 2004 [28] & Thesis & $5 / 12$ & $\begin{array}{c}\text { Adolescent, } 30 \% \\
\text { body fat } \uparrow\end{array}$ & M & 10 & ND & 10 & ND & BIA & $\begin{array}{l}\text { \% Body fat, Height, Lean mass, } \\
\text { Weight, WHR }\end{array}$ \\
\hline Jung HC, 2014 [30] & Thesis & $3 / 16$ & Adolescent & M & 15 & 13.9 & 15 & 13.9 & DXA & $\begin{array}{c}\text { \% Body fat, BMI, Fat mass, } \\
\text { Height, Lean mass, WC, Weight }\end{array}$ \\
\hline Kang MG, 2014 [31] & Thesis & $3 / 12$ & $\begin{array}{c}\text { Adolescent, } 30 \% \\
\text { body fat } \uparrow\end{array}$ & $\mathrm{F}$ & 10 & ND & 10 & $\mathrm{ND}$ & BIA & $\%$ Body fat, BMI, Weight \\
\hline Moon DS, 2007 [32] & Journal article & $5 / 12$ & $\begin{array}{l}\text { Elementary } \\
\text { student }\end{array}$ & $\mathrm{M}$ & 12 & 12.35 & 12 & 12.42 & BIA & $\begin{array}{l}\% \text { Body fat, BMI, Height, Lean } \\
\text { mass, Weight, WHR }\end{array}$ \\
\hline Kim WK, 2009 [33] & Journal article & $5 / 12$ & $\begin{array}{l}\text { Adolescent men, } \\
20 \% \text { body fat } \uparrow\end{array}$ & M & 10 & 14.7 & 10 & 15.1 & BIA & $\begin{array}{l}\text { \% Body fat, BMI, Height, Lean } \\
\text { mass, Weight, WHR }\end{array}$ \\
\hline Kwon YC, 2010 [34] & Journal article & $3 / 12$ & $\begin{array}{l}\text { Elementary } \\
\text { student, } 25 \% \\
\text { body fat } \uparrow\end{array}$ & Mixed & 12 & 11.92 & 12 & 12.5 & BIA & $\begin{array}{c}\text { \% Body fat, BMI, Fat mass, } \\
\text { Height, Lean mass, WC, Weight, } \\
\text { WHR }\end{array}$ \\
\hline Lee SH, 2011 [35] & Journal article & $4 / 10$ & Adolescent & M & 6 & 16.8 & 9 & 16.4 & BIA & $\begin{array}{l}\text { \% Body fat, Muscle mass, } \\
\text { Weight }\end{array}$ \\
\hline Cho WJ, 2013 [36] & Journal article & $3 / 12$ & $\begin{array}{l}\text { Elementary } \\
\text { student, BMI } \\
25 \mathrm{~kg} / \mathrm{m}^{2} \uparrow\end{array}$ & $\mathrm{M}$ & 12 & 11.17 & 12 & 11.33 & BIA & $\%$ Body fat, BMI, Weight \\
\hline
\end{tabular}


Table 1. Cont.

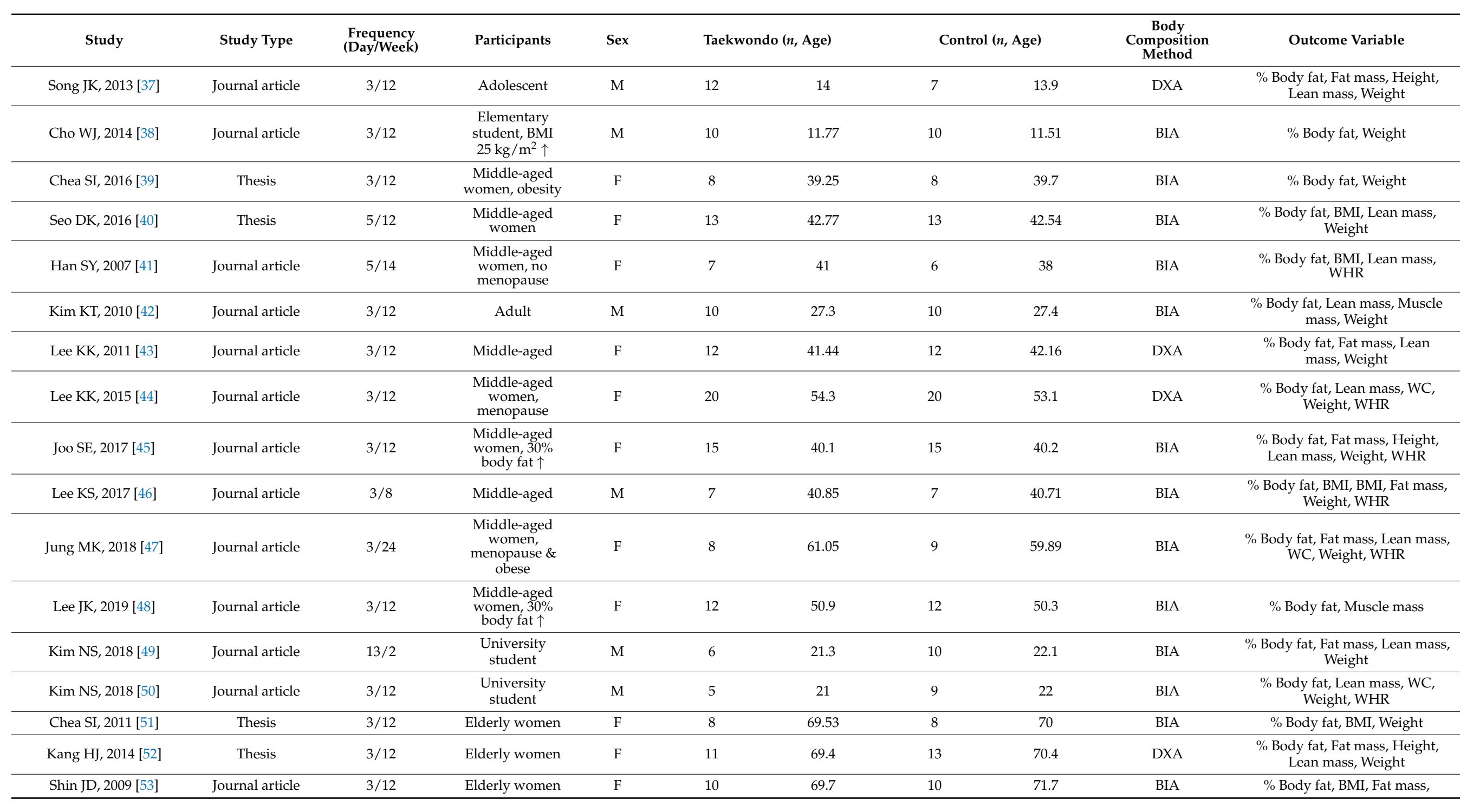


Table 1. Cont.

\begin{tabular}{|c|c|c|c|c|c|c|c|c|c|c|}
\hline \multirow{2}{*}{$\begin{array}{c}\text { Study } \\
\text { Moon DS, } 2010[54]\end{array}$} & \multirow{2}{*}{$\begin{array}{c}\text { Study Type } \\
\text { Journal article }\end{array}$} & \multirow{2}{*}{$\begin{array}{c}\begin{array}{c}\text { Frequency } \\
\text { (Day/Week) }\end{array} \\
3 / 12\end{array}$} & \multirow{2}{*}{$\begin{array}{c}\text { Participants } \\
\text { Elderly women }\end{array}$} & \multirow{2}{*}{$\begin{array}{l}\text { Sex } \\
F\end{array}$} & \multicolumn{2}{|c|}{ Taekwondo ( $n$, Age) } & \multicolumn{2}{|c|}{ Control $(n$, Age) } & \multirow{2}{*}{$\begin{array}{c}\begin{array}{c}\text { Body } \\
\text { Composition } \\
\text { Method }\end{array} \\
\text { BIA } \\
\end{array}$} & \multirow{2}{*}{$\begin{array}{c}\text { Outcome Variable } \\
\begin{array}{c}\% \text { Body fat, BMI, Height, Lean } \\
\text { mass, Weight }\end{array}\end{array}$} \\
\hline & & & & & 15 & 72.13 & 15 & 75.07 & & \\
\hline Cho WJ, 2012 [56] & Journal article & $3 / 12$ & $\begin{array}{l}\text { Elderly women, } \\
30 \% \text { body fat } \uparrow\end{array}$ & F & 13 & 69 & 13 & 68.62 & BIA & $\%$ Body fat, Weight \\
\hline Lim YR, 2017 [57] & Journal article & $4 / 12$ & $\begin{array}{l}\text { Elderly women, } \\
\text { over Weight }\end{array}$ & $\mathrm{F}$ & 15 & ND & 15 & ND & BIA & $\begin{array}{c}\% \text { Body fat, Fat mass, Muscle } \\
\text { mass, Weight }\end{array}$ \\
\hline
\end{tabular}




\subsubsection{Weight}

A total of 34 studies reported body weight (total $n=738$; taekwondo training group $n=365$, control group $n=373$ ). The mean difference (MD) in body weight between the Taekwondo training and the control group was -3.085 (95\% CI; $-3.720,-2.449)$. That is, the weight of the Taekwondo training group decreased by $3.1 \mathrm{~kg}$ compared to the control group, which was statistically significant $(p<0.001)$. For the heterogeneity test, the results of Cochrane $\mathrm{Q}$ were $p=0.59$, and Higgins' $\mathrm{I}^{2}$ was $0 \%$ (Figure $3 \mathrm{~b}$ ).

\subsubsection{Body Mass Index}

Eighteen studies reported body mass index (total $n=395 ; 198$ Taekwondo training group, 197 control group). The mean difference (MD) of body mass index between the Taekwondo training and the control group was -1.503 (95\% CI; $-1.803,-1.204)$. The Body mass index of the Taekwondo training group decreased by 1.5 compared to the control group, and there was a statistically significant difference $(p<0.001)$. For the heterogeneity test, the results of Cochrane $\mathrm{Q}$ were $p=0.82$, and Higgins' $\mathrm{I}^{2}$ was $0 \%$ (Figure $3 c$ ).

\subsubsection{Waist Circumference}

Six studies reported waist circumference (total $n=136$; taekwondo training group $n=66$, control group $n=70$ ). The mean difference (MD) in waist circumference between the Taekwondo training and the control group was -2.231 (95\% CI; $-3.855,-0.607)$. The waist circumference of the Taekwondo training group decreased by $2.2 \mathrm{~cm}$ compared to the control group, and there was a statistically significant difference $(p<0.05)$. For the heterogeneity test, the results of Cochrane Q were $p=0.81$, and Higgins' $\mathrm{I}^{2}$ was $0 \%$ (Figure 3d).

\subsubsection{Waist-Hip Ratio}

Ten studies reported the waist-hip ratio (total $n=215$; Taekwondo training group $n=105$, control group $n=110$ ). The mean difference (MD) in the waist-hip ratio between the Taekwondo training and the control groups was -0.029 (95\% CI; $-0.015,-0.010)$. The waist-hip ratio of the Taekwondo training group decreased by 0.03 compared to the control group, and there was a statistically significant difference $(p<0.05)$. For the heterogeneity test, the results of Cochrane $\mathrm{Q}$ were $p<0.01$, and Higgins' $\mathrm{I}^{2}$ was $0 \%$ (Figure $3 \mathrm{e}$ ).

\subsubsection{Body Fat Mass}

Fourteen studies reported body fat mass (total $n=327$; Taekwondo training group $n=164$, the control group $n=163$ ). The average difference (MD) in body fat mass between the Taekwondo training and the control group was -1.398 (95\% CI; $-2.109,-0.687$ ). The body fat mass of the Taekwondo training group decreased by $1.40 \mathrm{~kg}$ compared to the control group, and there was a statistically significant difference $(p<0.01)$. For the heterogeneity test, the results of Cochrane $\mathrm{Q}$ were $p=0.46$, and Higgins' $\mathrm{I}^{2}$ was $0 \%$ (Figure 3f).

\subsubsection{Body Fat Percentage}

Thirty-six studies reported body fat percentage (total $n=750$; taekwondo training group $n=371$, control group $n=379$ ). The mean difference (MD) in body fat percentage between the Taekwondo training and the control group was $-2.850(95 \% \mathrm{CI} ;-3.327$, $-2.373)$. The body fat percentage of the Taekwondo training group decreased by $-2.90 \%$ compared to the control group, and there was a statistically significant difference $(p<0.001)$. For the heterogeneity test, the results of Cochrane $\mathrm{Q}$ were $p=0.23$, and Higgins ${ }^{\prime} \mathrm{I}^{2}$ was $14 \%$ (Figure $3 g$ ).

\subsubsection{Lean Mass}

Twenty-four studies reported lean mass (total $n=531$; taekwondo training group $n=263$, control group $n=268$ ). The average difference (MD) in lean mass between the 
Taekwondo training and the control groups was 1.109 (95\% CI; 0.366, 1.851). The lean mass of the Taekwondo training group increased by $1.11 \mathrm{~kg}$ compared to the control group, and there was a statistically significant difference $(p<0.05)$. For the heterogeneity test, the results of Cochrane $\mathrm{Q}$ were $p=0.09$, and Higgins' $\mathrm{I}^{2}$ was $30 \%$ (Figure $3 \mathrm{~h}$ ).

\subsubsection{Muscle Mass}

Seven studies reported muscle mass (total $n=170$; Taekwondo training group $n=84$, control group $n=86$ ). The mean difference (MD) in muscle mass between the Taekwondo training and the control group was 1.818 (95\% CI; 0.208, 3.427). The muscle mass of the Taekwondo training group increased by $1.82 \mathrm{~kg}$ compared to the control group, and there was a statistically significant difference $(p<0.05)$. For the heterogeneity test, the results of Cochrane $\mathrm{Q}$ were $p=0.01$, and Higgins' $\mathrm{I}^{2}$ was $63 \%$ (Figure $3 \mathrm{i}$ ).

\subsection{Moderator Analyses}

The results of meta-ANOVA with categorical variables (gender, age, study period, publication type) are shown in Tables 2 and 3. No statistical significance was reported on moderating variables of height, WC, WHR, body fat mass, lean mass, and muscle mass. Meanwhile, in the case of body weight $(p=0.044)$, BMI $(p=0.036)$, and body fat percentage $(p=0.026)$, the age group was confirmed as a statistically significant moderating variable. It was confirmed that the decrease rate of weight and BMI was the highest among the student group, and the decrease rate of body fat percentage was highest among the elderly group.

\subsection{Publication Bias}

Funnel plots were used to analyze publication bias or effects of small-sized research, as shown in Figure 4. No publication bias was found from Egger's linear regression test among all variables except weight and body fat percentage, excluding small-sized studies (WC, muscle mass). Also, as a result of Begg's test using a rank correlation test, no factor showed a significant difference, so the publication bias of the literature used in this study could not be confirmed. 
Table 2. Effects of moderators on height, weight, BMI, WC, WHR.

\begin{tabular}{|c|c|c|c|c|c|c|c|c|c|c|c|c|c|c|c|c|c|c|c|c|c|c|c|c|c|}
\hline \multirow{3}{*}{\begin{tabular}{l}
\multicolumn{1}{c}{ Variables } \\
Sex \\
Male \\
Female
\end{tabular}} & \multicolumn{5}{|c|}{ Height } & \multicolumn{5}{|c|}{ Weight } & \multicolumn{5}{|c|}{ BMI } & \multicolumn{5}{|c|}{ Waist Circumference } & \multicolumn{5}{|c|}{ WHR } \\
\hline & \multirow[b]{2}{*}{$\begin{array}{l}9 \\
5\end{array}$} & \multirow{2}{*}{$\begin{array}{l}\text { MD } \\
\\
0.246 \\
0.271\end{array}$} & \multicolumn{2}{|c|}{$95 \% \mathrm{CI}$} & \multirow{2}{*}{$\begin{array}{c}p^{+} \\
0.985\end{array}$} & \multirow{2}{*}{$\begin{array}{c}k \\
18 \\
14\end{array}$} & \multirow{2}{*}{$\begin{array}{l}\text { MD } \\
-3.314 \\
-2.147\end{array}$} & \multicolumn{2}{|c|}{$95 \% \mathrm{CI}$} & \multirow{2}{*}{$\begin{array}{c}p^{\dagger} \\
0.154\end{array}$} & \multirow{2}{*}{$\begin{array}{l}k \\
9 \\
8\end{array}$} & \multirow{2}{*}{$\begin{array}{l}\text { MD } \\
-1.708\end{array}$} & \multicolumn{2}{|c|}{$95 \% \mathrm{CI}$} & \multirow{2}{*}{$\begin{array}{l}p^{+} \\
0.100\end{array}$} & \multirow{2}{*}{$\begin{array}{l}k \\
2\end{array}$} & \multirow{2}{*}{$\begin{array}{l}\text { MD } \\
-4.063 \\
-1421\end{array}$} & \multicolumn{2}{|c|}{$95 \% \mathrm{CI}$} & \multirow{2}{*}{$\begin{array}{l}p^{+} \\
0.404\end{array}$} & \multirow{2}{*}{$\begin{array}{l}k \\
5 \\
4\end{array}$} & \multirow{2}{*}{$\begin{array}{l}\text { MD } \\
-0.045 \\
-0.015\end{array}$} & \multicolumn{2}{|c|}{$95 \% \mathrm{CI}$} & \multirow{2}{*}{$\begin{array}{c}p^{+} \\
0.111\end{array}$} \\
\hline & & & $\begin{array}{l}-1.412 \\
-1.774\end{array}$ & $\begin{array}{l}1.905 \\
2.136\end{array}$ & & & & $\begin{array}{l}-4.171 \\
-3.505\end{array}$ & $\begin{array}{l}-2.455 \\
-0.790\end{array}$ & & & & $\begin{array}{l}-2.074 \\
-1.738\end{array}$ & $\begin{array}{l}-1.342 \\
-0.399\end{array}$ & & & & $\begin{array}{l}-9.870 \\
-3.605\end{array}$ & $\begin{array}{l}1.743 \\
0.762\end{array}$ & & & & $\begin{array}{l}-0.078 \\
-0.031\end{array}$ & $\begin{array}{l}-0.012 \\
-0.001\end{array}$ & \\
\hline $\begin{array}{l}\text { Study duration } \\
\leq 12 \text { weeks } \\
>12 \text { weeks }\end{array}$ & $\begin{array}{l}14 \\
2\end{array}$ & $\begin{array}{l}0.297 \\
-0.012\end{array}$ & $\begin{array}{l}-1.001 \\
-4.556\end{array}$ & $\begin{array}{l}1.595 \\
4.531\end{array}$ & 0.898 & $\begin{array}{c}31 \\
3\end{array}$ & $\begin{array}{l}-3.107 \\
-2.315\end{array}$ & $\begin{array}{l}-3.753 \\
-6.143\end{array}$ & $\begin{array}{l}-2.462 \\
1.513\end{array}$ & 0.689 & $\begin{array}{l}16 \\
3\end{array}$ & $\begin{array}{l}-1.465 \\
-1.731\end{array}$ & $\begin{array}{l}-1.789 \\
-2.521\end{array}$ & $\begin{array}{l}-1.141 \\
-0.941\end{array}$ & 0.542 & $\begin{array}{l}5 \\
1\end{array}$ & $\begin{array}{l}-2.059 \\
-5.000\end{array}$ & $\begin{array}{l}-3.732 \\
-11.719\end{array}$ & $\begin{array}{c}-0.386 \\
1.719\end{array}$ & 0.405 & 10 & -0.029 & -0.048 & -0.010 & - \\
\hline
\end{tabular}

MD-Mean difference by the Hedges. $k$-number of observations. ${ }^{\dagger} p$-value of meta-ANOVA for categorical moderators.

Table 3. Effects of moderators on fat mass, \% fat, lean mass, muscle mass.

\begin{tabular}{|c|c|c|c|c|c|c|c|c|c|c|c|c|c|c|c|c|c|c|c|c|}
\hline \multirow{3}{*}{\begin{tabular}{l}
\multicolumn{1}{c}{ Variables } \\
Sex \\
Male \\
Female
\end{tabular}} & \multicolumn{5}{|c|}{ Fat Mass } & \multicolumn{5}{|c|}{ \% Body Fat } & \multicolumn{5}{|c|}{ Lean Mass } & \multicolumn{5}{|c|}{ Muscle Mass } \\
\hline & \multirow{2}{*}{$\begin{array}{l}k \\
\\
6 \\
7\end{array}$} & \multirow{2}{*}{$\begin{array}{c}\text { MD } \\
\\
-1.043 \\
-0.777\end{array}$} & \multicolumn{2}{|c|}{$95 \%$ CI } & \multirow{2}{*}{$\begin{array}{c}p^{+} \\
0.761\end{array}$} & \multirow{2}{*}{$\begin{array}{l}k \\
17 \\
17\end{array}$} & \multirow{2}{*}{$\begin{array}{l}\text { MD } \\
-2.641 \\
-3.006 \\
\end{array}$} & \multicolumn{2}{|c|}{$95 \%$ CI } & $\begin{array}{c}p^{+} \\
0.404\end{array}$ & \multirow{2}{*}{$\begin{array}{l}k \\
12 \\
10\end{array}$} & \multirow{2}{*}{$\begin{array}{c}\text { MD } \\
\\
1.718 \\
0.364\end{array}$} & \multicolumn{2}{|c|}{$95 \%$ CI } & $\begin{array}{c}p^{+} \\
0.088\end{array}$ & \multirow{2}{*}{$\begin{array}{l}k \\
5 \\
2\end{array}$} & \multirow{2}{*}{\begin{tabular}{c|} 
MD \\
1.733 \\
2.031
\end{tabular}} & \multicolumn{2}{|c|}{$95 \%$ CI } & \multirow{2}{*}{$\begin{array}{c}p^{+} \\
0.896\end{array}$} \\
\hline & & & $\begin{array}{l}-2.480 \\
-1.717\end{array}$ & $\begin{array}{l}0.393 \\
0.163 \\
\end{array}$ & & & & $\begin{array}{l}-3.228 \\
-3.556\end{array}$ & $\begin{array}{l}-2.121 \\
-2.457\end{array}$ & & & & $\begin{array}{c}0.388 \\
-0.440\end{array}$ & $\begin{array}{l}3.048 \\
1.169\end{array}$ & & & & $\begin{array}{l}-0.125 \\
-2.056\end{array}$ & $\begin{array}{l}3.59 \\
6.117\end{array}$ & \\
\hline $\begin{array}{l}\text { Study duration } \\
\leq 12 \text { weeks } \\
>12 \text { weeks }\end{array}$ & $\begin{array}{c}13 \\
1\end{array}$ & $\begin{array}{l}-1.370 \\
-2.400\end{array}$ & $\begin{array}{l}-2.134 \\
-6.265\end{array}$ & $\begin{array}{c}-0.606 \\
1.465\end{array}$ & 0.610 & $\begin{array}{c}33 \\
3\end{array}$ & $\begin{array}{l}-2.756 \\
-3.220\end{array}$ & $\begin{array}{l}-3.298 \\
-4.375\end{array}$ & $\begin{array}{l}-2.213 \\
-2.065\end{array}$ & 0.475 & $\begin{array}{c}23 \\
1\end{array}$ & $\begin{array}{c}1.173 \\
-4.300\end{array}$ & $\begin{array}{c}0.447 \\
-11.207\end{array}$ & $\begin{array}{l}1.900 \\
2.607\end{array}$ & 0.122 & 7 & 1.818 & $\begin{array}{c}0.208 \\
-\end{array}$ & 3.427 & \\
\hline $\begin{array}{l}\text { Publication type } \\
\text { Thesis } \\
\text { Journal }\end{array}$ & $\begin{array}{l}5 \\
9\end{array}$ & $\begin{array}{l}-1.039 \\
-1.572\end{array}$ & $\begin{array}{l}-2.283 \\
-2.438\end{array}$ & $\begin{array}{c}0.205 \\
-0.706\end{array}$ & 0.544 & $\begin{array}{l}14 \\
22\end{array}$ & $\begin{array}{l}-2.673 \\
-2.923\end{array}$ & $\begin{array}{l}-3.457 \\
-3.551\end{array}$ & $\begin{array}{l}-1.889 \\
-2.295\end{array}$ & 0.627 & $\begin{array}{l}15 \\
19\end{array}$ & $\begin{array}{l}-3.775 \\
-2.739\end{array}$ & $\begin{array}{l}-4.874 \\
-3.518\end{array}$ & $\begin{array}{l}-2.675 \\
-1.960\end{array}$ & 0.132 & $\begin{array}{l}3 \\
4\end{array}$ & $\begin{array}{l}1.854 \\
1.742\end{array}$ & $\begin{array}{l}-0.984 \\
-0.522\end{array}$ & $\begin{array}{l}4.692 \\
4.007\end{array}$ & 0.952 \\
\hline
\end{tabular}

MD-Mean difference by the Hedges. $k$-number of observations. ${ }^{\dagger} p$-value of meta-ANOVA for categorical moderators. 

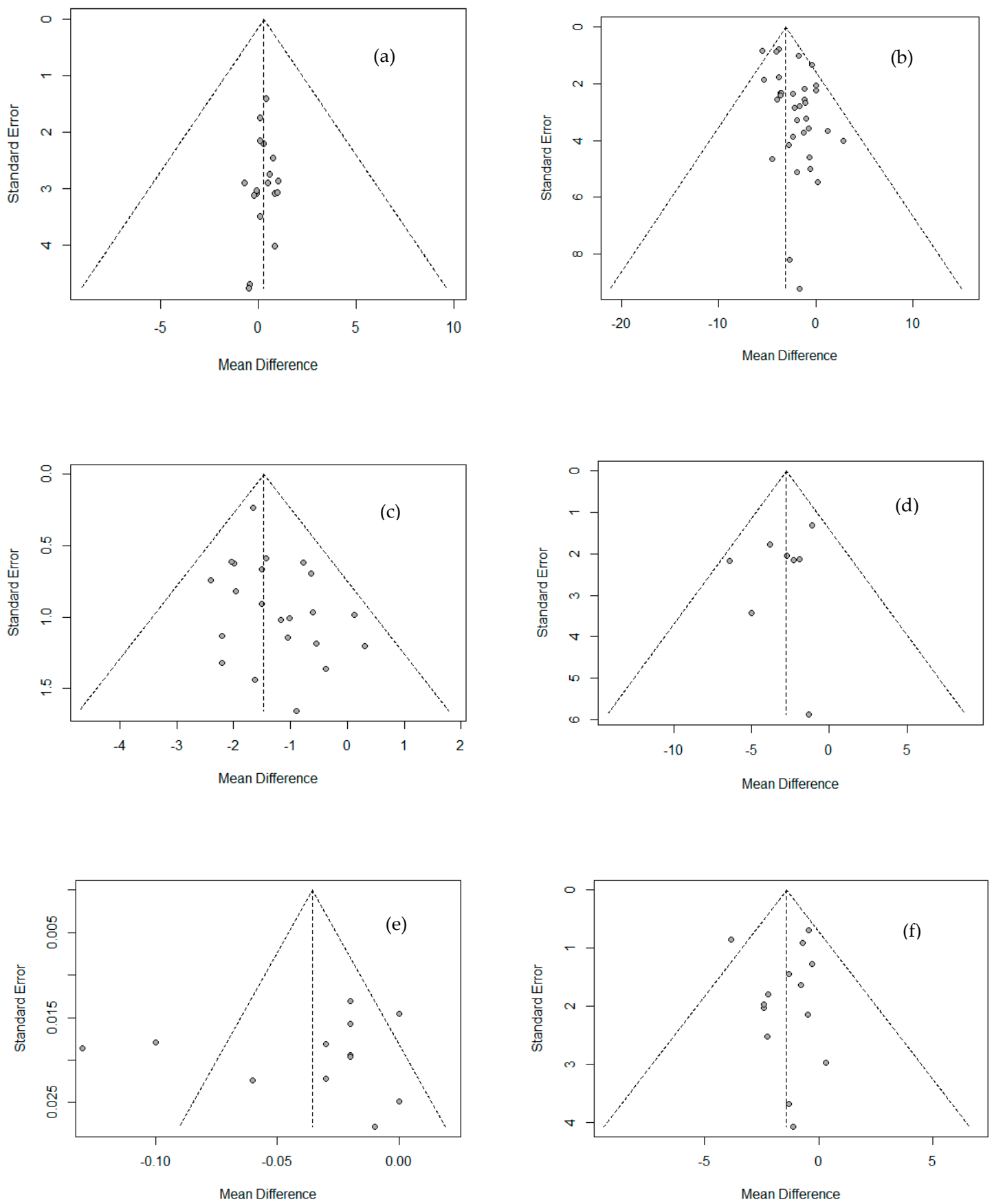

Figure 4. Cont. 

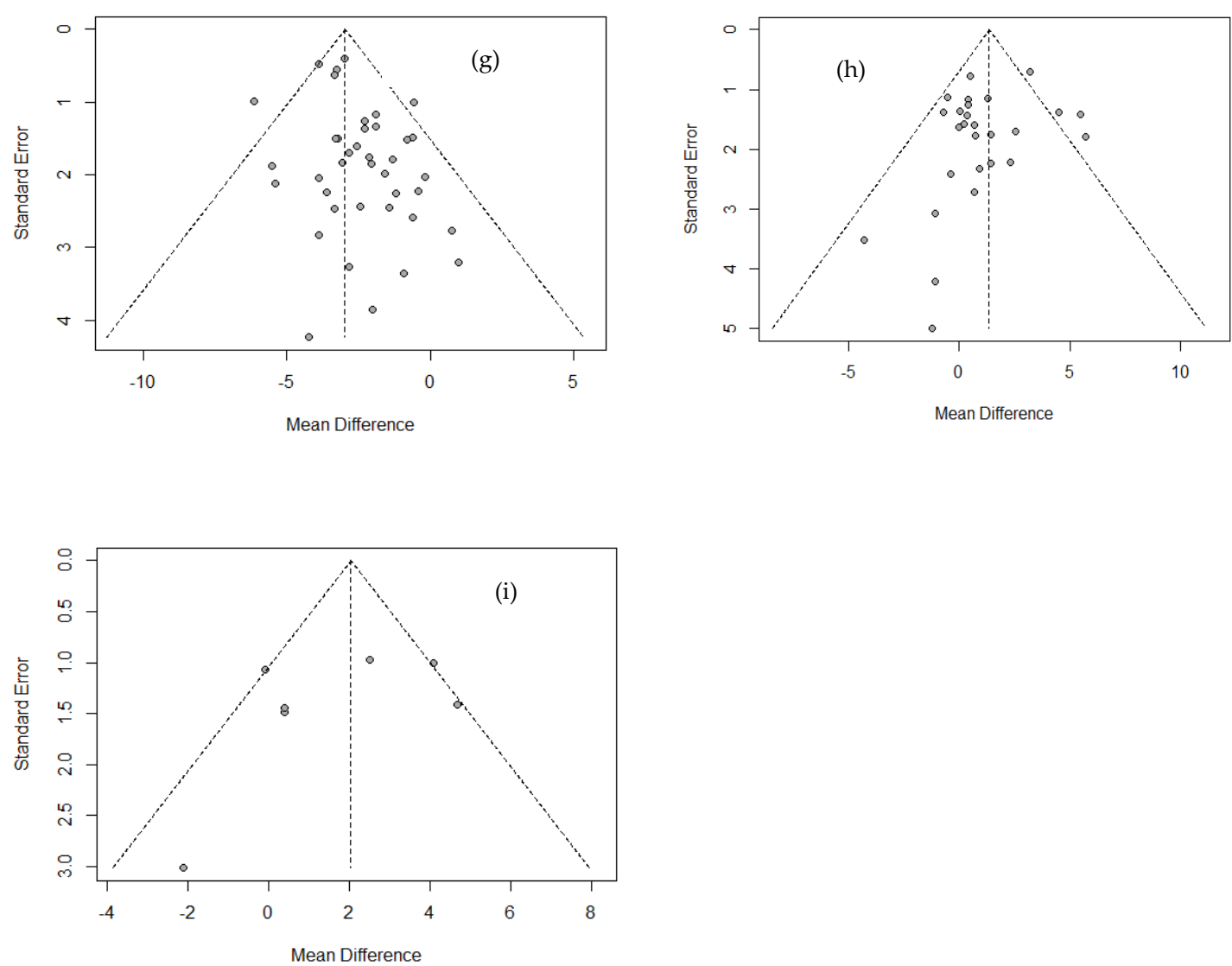

Figure 4. Funnel plot showing the publication bias of Physique and body composition. (a) Height; (b) weight; (c) BMI; (d) WC; (e) WHR; (f) fat mass.; (g) \% body fat; (h) lean mass; (i) muscle mass.

\section{Discussion}

Since changes in body composition occur throughout the lifespan due to growth, maturation and aging, as well as factors such as diseases and behaviors, it is a representative indicator for determining the level of body development [58]. Observing changes in body composition is an important factor in determining health, disease, exercise, and nutrition. It is also a very important factor in physique and athletic performance [59].

In general, Taekwondo training in Korea consists of 5 sessions per week, $1 \mathrm{~h}$ per session [60]. The training period is about 12 weeks, which we present in Table 1 . Taekwondo exercise consists of $5 \mathrm{~min}$ each of warm-up and cool-down routines, followed by $50 \mathrm{~min}$ of exercise including basic Taekwondo movements, Poomsae, and Gyeorugi (fighting simulation) as the main exercises. Most taekwondo training centers apply a similar method. Therefore, the effect of taekwondo training in this study has very suitable conditions for meta-analysis.

As a result of analyzing the effect of Taekwondo training on changes in body composition, statistically significant differences were found in body weight, BMI, WC, body fat mass, body fat percentage, lean mass, and muscle mass, excluding height. Especially in the case of students, body weight and body fat percentage decreased significantly, and lean mass and muscle mass also tended to increase. In the elderly, a significant decrease in body fat percentage and an increase in muscle mass were found.

According to ACSM guidelines, exercise intensity $40-70 \% \mathrm{VO}_{2} \mathrm{R}$ (oxygen uptake reserve) is recommended for overweight and obesity management [61]. Taekwondo's basic movements, kicks, and Poomsae movements have an exercise intensity of $84 \%$ HRmax and 56.8 82.2\% $\mathrm{VO}_{2} \max$ [62]. Taekwondo competition is a high-intensity exercise with an exercise intensity of 10 METs [63]. As such, Taekwondo exhibits anaerobic exercise patterns 
during matches and competitions and aerobic exercise patterns during taekwondo aerobics, Poomsae, sparring steps, basic movements, and moving kicks.

Therefore, the results of this study are clear evidence that the type of exercise and intensity of Taekwondo has positive effects on reducing body weight and body fat and increasing lean mass. In addition, there is a disadvantage that long and tedious exercise time is required to obtain meaningful exercise effects with general aerobic exercise such as walking or running [64]. In contrast, Taekwondo is an interval training type that alternates high- and low-intensity exercise repeatedly. In addition, Taekwondo gives interest and has higher exercise effects within the same amount of exercise time than other exercise types such as walking or moderate running [65]. Therefore, the exercise program using Taekwondo will positively improve obesity, the most severe health issue in Korea, and contribute significantly to preventing chronic diseases such as cardiovascular disease.

We found one unfortunate thing while conducting this study. That is, many studies reporting on the effectiveness of Taekwondo training do not apply an RCT design or even have no control group. In fact, there were many documents excluded from the analysis because there was no control group during this study. Therefore, if researchers who want to explain the effects of Taekwondo training in the future follow the RCT design well, follow-up researchers will find clearer results.

\section{Conclusions}

We found that taekwondo training at a frequency of five times per week for more than 12 weeks positively improved the obesity factor through this study. These findings are clear evidence that Taekwondo training is an effective exercise that can prevent or positively improve obesity. In addition, it shows that Taekwondo has value as a lifestyle sport that can contribute to the promotion of human health, not just bounded in the field of martial arts and sports.

Author Contributions: Conceptualization, S.B. and S.-S.N.; methodology, S.B., J.-D.L. and S.-S.N.; software, S.B.; validation, S.B., J.-B.P. and S.-S.N.; formal analysis, S.B. and S.-S.N.; investigation, S.B., S.-H.C. and J.-B.P.; resources, S.B. and J.-D.L.; data creation, S.B. and J.-D.L.; writing original draft preparation, S.B. and S.-S.N.; writing-review and editing, S.B., J.-D.L. and S.-S.N.; visualization, S.B.; supervision, S.-H.C., J.-B.P. and S.-S.N.; project administration, S.B. and S.-S.N.; funding acquisition, J.-D.L., S.-H.C., J.-B.P. and S.-S.N. All authors have read and agreed to the published version of the manuscript.

Funding: This research was supported by Taekwondo Research Institute of Kukkiwon.

Institutional Review Board Statement: The study was conducted according to the guidelines of the Declaration of Helsinki and approved by the Ethics Committee of SungShin Women's University SSWUIRB-2021-030 and 06-12-2021).

Informed Consent Statement: Not applicable.

Data Availability Statement: Not applicable.

Acknowledgments: All authors sincerely thank Kukkiwon President Lee Dongsub for supporting this study.

Conflicts of Interest: The authors declare no conflict of interest.

\section{References}

1. World Taekwondo Federation. Vision, Mission, Strategy; World Taekwondo Federation: Seoul, Korea, 2021. Available online: http:/ /www.worldtaekwondo.org/about-wt/about.html (accessed on 10 August 2021).

2. Ministry of Health and Welfare. The 3rd National Health Plan (2011-2020); Ministry of Health and Welfare: Seoul, Korea, 2011.

3. Korea Center for Disease Control Trend of Physical Activity Practice Rate, 2007-2017. 2019. Available online: http:/ /www.kdca. go.kr/filepath /boardDownload.es?bid=0034\&list_no=364483\&seq=1 (accessed on 10 August 2021).

4. Guthold, R.; Stevens, G.A.; Riley, L.M.; Bull, F.C. Global trends in insufficient physical activity among adolescents: A pooled analysis of 298 population-based surveys with 1.6 million participants. Lancet Child. Adolesc. Health 2020, 4, 23-35. [CrossRef] 
5. Powers, S.K.; Smuder, A.J.; Criswell, D.S. Mechanistic links between oxidative stress and disuse muscle atrophy. Antioxid. Redox Signal. 2011, 15, 2519-2528. [CrossRef]

6. Swift, D.L.; Johannsen, N.M.; Lavie, C.J.; Earnest, C.P.; Church, T.S. The role of exercise and physical activity in weight loss and maintenance. Prog. Cardiovasc. Dis. 2014, 56, 441-447. [CrossRef]

7. Byun, J.C. body composition, Cholesterol, Lumbar and femur BMD and bone marker hormones by Taekwondo training in children. Korean J. Growth Dev. 2005, 13, 41-51.

8. Song, E. The Effects of a 10-Week Taekwon Diet Program on Body Composition, Blood Pressure, Blood Glucose and Physical Self-Description of Obese Women. Ph.D. Thesis, Kyung Hee University, Yongin, Korea, 2018.

9. Lee, D.M. Effects of Taekwondo training on left ventricular function and cardiovascular disease risk factor in hypertensive obese elderly women. Ph.D. Thesis, Dong-A University, Busan, Korea, 2013.

10. Kim, S.B. The effects of Poomsae training of Taekwondo on senile demantia factor and physical fitness in the elderly. Master's Thesis, Dong-A University, Busan, Korea, 2009.

11. Marković, G.; Mišigoj-Duraković, M.; Trninić, S. Fitness profile of elite Croatian female taekwondo athletes. Coll. Antropol. 2005, 29, 93-99. [PubMed]

12. Heller, J.; Peric, T.; Dlouha, R.; Kohlikova, E.; Melichna, J.; Novakova, H. Physiological profiles of male and female taekwon-do (ITF) black belts. J. Sports Sci. 1998, 16, 243-249. [CrossRef]

13. Bouhlel, E.; Jouini, A.; Gmada, N.; Nefzi, A.; Abdallah, K.B.; Tabka, Z. Heart rate and blood lactate responses during Taekwondo training and competition. Sci. Sports 2006, 21, 285-290. [CrossRef]

14. Hwang, S.D. Easy-to-Understand Meta-Analysis; Hakjisa Publishing: Seoul, Korea, 2014.

15. Moher, D.; Shamseer, L.; Clarke, M.; Ghersi, D.; Liberati, A.; Petticrew, M.; Shekelle, P.; Stewart, L.A. Preferred reporting items for systematic review and meta-analysis protocols (PRISMA-P) 2015 statement. Syst. Rev. 2015, 4, 1-9. [CrossRef] [PubMed]

16. Higgins, J.P.; Thomas, J.; Chandler, J.; Cumpston, M.; Li, T.; Page, M.J.; Welch, V.A. Cochrane Handbook for Systematic Reviews of Interventions; John Wiley \& Sons: Chichester, UK, 2019. [CrossRef]

17. Shim, S.R.; Kim, S. Intervention meta-analysis: Application and practice using R software. Epidemiol. Health 2019, 41 , e2019008. [CrossRef] [PubMed]

18. DerSimonian, R.; Laird, N. Meta-analysis in clinical trials. Control. Clin. Trials 1986, 7, 177-188. [CrossRef]

19. Shim, S.R. R Meta-Analysis for Medical and Health Researchers; Hannarae Publishing: Seoul, Korea, 2019.

20. Shim, S.R.; Shin, I.S.; Bae, J.M. Intervention meta-analysis using STATA software. J. Health Inform. Stat. 2016, 41, 123-134. [CrossRef]

21. Choi, W. The Effect of Taekwondo Training Activity on the Development of Physical Strength toward Obesity Children. Master's Thesis, Dong-A University, Busan, Korea, 2000.

22. Lee, S.H. Effect of Taekwondo Program and Calcium Intake on Body Composition, Physical Fitness, Growth Hormone, and IGF-1 in Elementary School Male Students. Master's Thesis, Pusan National University, Busan, Korea, 2008.

23. Lee, S.W. Effects of Taekwondo Training on Physical Fitness and Immune System Function in Infantile Obesity. Master's Thesis, Pusan National University, Busan, Korea, 2011.

24. Jeong, S.H. Effect of Official Rhythmic Poomsae Training Program on Body Composition, Physical Fitness and Growth Factor in Elementary Students. Master's Thesis, Dong-A University, Busan, Korea, 2012.

25. Lee, S.J. The Effect of Taekwondo Training on Body Composition, Physical Fitness, and Balance Ability in Obese Grade-Schooler. Master's Thesis, Chosun University, Gwangju, Korea, 2014.

26. Lee, S.H. The effects of Taekwondo training on PAPS, Growth Factor and Attention concentration in Children. Master's Thesis, Dong-A University, Busan, Korea, 2015.

27. Seo, D.W. Effect of Taekwondo Training on Body Composition, Bone Mineral Density and Isokinetic Concentric/Eccentric Contractions of Teenagers for 12 Weeks. Ph.D. Thesis, Woosuk University, Wanju-gun, Korea, 2019.

28. An, S.W. The Effects of Taekwondo Training on Physical Fitness and Insulin in Obese Adolescents. Master's Thesis, Dong-A University, Busan, Korea, 2004.

29. Seo, D.K. The Effect of Taekwondo Training on Body Composition, Physical Fitness and Growth Factors in Female Students after Menarche. Master's Thesis, Dong-A University, Busan, Korea, 2008.

30. Jung, H.C. Effects of 16 Weeks of Taekwondo Training on Abdominal Fat, Adipocytokines, Bone Mineral Density, Bone Turnover Markers, and Health-Related Fitness in Obese Male Adolescents. Ph.D. Thesis, Kyunghee University, Yongin, Korea, 2014.

31. Kang, M.G. The Effects of Taekwondo Training on Body Composition, Blood Lipid, and Adiponectin in Obese Middle School Girls. Ph.D. Thesis, Chosun University, Gwangju, Korea, 2014.

32. Moon, D.S.; Kim, D.Y. The Effect of Taekwondo on Body Composition, Physical Fitness and Growth Factors in Elderly Women. Korea Sport Res. 2007, 18, 495-506.

33. Kim, W.K.; Kwon, Y.C. The Effect of Taekwondo Training on Physical Fitness and Growth Hormone, IGF-1 or DHEAs Concentration in Obesity Adolescent. Korean J. Sports Sci. 2009, 18, 1007-1018.

34. Kwon, Y.C.; Park, S.K.; Kim, E.H.; Park, J.K.; Jang, J.H. Effects of Taekwondo Training on Body Composition, Physical Fitness and Serum Adiponectin in Children Obesity. J. Korean Alliance Martial Arts 2010, 12, 239-251. [CrossRef]

35. Lee, S.H.; Baek, Y.H. The Effect of Taekwondo and Allium Tuberosum Intake on Body Composition, Blood Lipids and C-Reactive Protein. J. Life Sci. 2011, 21, 265-272. [CrossRef] 
36. Cho, W.J.; Jeoung, J.H. Effects of Taekwondo Poomsae Training on Body Composition, Blood Lipid, and Adiponectin in Obese Children. J. Korean Alliance Martial Arts 2013, 15, 57-68. [CrossRef]

37. Song, J.K.; Kim, H.B.; Kang, H.J.; Jung, H.C. Effect of 12 weeks Taekwondo Poomsae training on body composition, health-related fitness and dietary intake in male adolescents. Taekwondo J. Kukkiwon 2013, 4, 61-76. [CrossRef]

38. Cho, W.J.; Yoon, O.N. The Effect of 12Weeks Taekwondo Training Program on Blood Lipid and Growth Hormone in Obese Children. Korean J. Growth Dev. 2014, 22, 267-272.

39. Chea, S.I. Effects on Lipid Metabolic-related Hormones, Inflammatory Markers and Metabolic Syndrome of Intermittent High Intensity Interval Training Using Taekwondo and Continuous Aerobic Training in Middle-Aged Obese Women. Ph.D. Thesis, Hanyang University, Ansan, Korea, 2016.

40. Seo, D.K. The Effect of Taekwondo Exercise on Body Composition, Neurotransmitter and Brain Nerve Growth Factor in Middle-aged Women. Ph.D. Thesis, Pukyong National University, Busan, Korea, 2016.

41. Han, S.Y.; Kim, H.; Lee, S.H. Effects of Basic Taekwondo Movement Training on Body Composition, Cardiorespiratory of Middle-aged Women. Korean J. Sports Sci. 2007, 16, 667-681.

42. Kim, K.T. Influence of Taekwondo and Combined Strength Exercise on Health-Related Physical Fitness and Blood Lipid in Private Security Guards. J. Korean Alliance Martial Arts 2010, 12, 265-276. [CrossRef]

43. Lee, K.K. The Effect of Taekwondo Training on Electrophysiological Stress, Stress Hormone and Body Composition (BIA \& CT) in Middle-Aged Women. Korean J. Sports Sci. 2011, 20, 1029-1039.

44. Lee, K.K. Effects of Taekwondo Training on Body Composition, Blood Lipid and Bone Mineral Density in Postmenopausal Women. J. Sport Leis. Stud. 2015, 61, 539-547. [CrossRef]

45. Joo, S.E.; Jung, H.C.; Kang, H.J.; Jung, S.W.; Seo, M.W.; Kim, S.W.; Song, J.K. Effect of 12 weeks of Taekwondo training on body fatness, health-related fitness and isokinetic muscle strength in obese women aged 35-55 years. Korean Soc. Sports Sci. 2017, 26, 879-891. [CrossRef]

46. $\quad$ Lee, K.S.; An, Y.J.; Kim, T.W.; Son, H.J.; Yang, J.H.; Mo, K.W.; Kim, S.K.; Jang, C.H. The Effect of 8 Weeks Rope-Skipping Exercise and Taekwondo Exercise on Blood lipids, Vascular Compliance, Oxidant Stress and Antioxidant Capacity in Middle Aged Men. Korean J. Phys. Educ. 2017, 56, 651-663. [CrossRef]

47. Jeong, M.K.; Ryu, J.K.; Jung, H.H.; Kim, Y.H.; Park, S.K. Effects of Taekwondo aerobic and Combined Exercise Program on Health-related Physical Fitness and Physical Activity and Depression Scale in Menopausal Obesity Women. Korean Soc. Sports Sci. 2018, 27, 1199-1210. [CrossRef]

48. Lee, J.G.; Lee, S.H. Effects of Taekwondo Exercise on Body Composition, Lipid Profile and Leptin in Obese Middle-aged Women. J. World Soc. Taekwondo Cult. 2019, 10, 147-159. [CrossRef]

49. Kim, N.S.; Lee, J.Y. The Effect of 12 weeks Taekwondo Training on Body-Composition, Blood Irisin, Adiponectin, and Lipids Levels in Obese College Students. Korean Soc. Sports Sci. 2018, 27, 1081-1091. [CrossRef]

50. Kim, N.S.; Lee, J.Y. The Effect of Long-Term Taekwondo Participation on Blood Glucose, Insulin, CRP levels, HOMA-IR, and Body-Composition in Obese Male College Students. Korean J. Sports Sci. 2018, 27, 1011-1019. [CrossRef]

51. Chea, S.I. A Study on the Effect of Functional Fitness, Body composition and Vascular Compliance before and after 12 weeks Taekwondo Training in Elder Women. Master's Thesis, Hanyang University, Ansan, Korea, 2011.

52. Kang, H.J. Effects of 12 Weeks of Taekwondo and Resistance Training on Bone Mineral Density, Bone Turnover Markers, Functional Fitness, and Arterial Compliance in Elderly Women. Ph.D. Thesis, Kyunghee University, Yongin, Korea, 2014.

53. Shin, J.D.; Kim, W.K. Effects of taekwondo poomsae training on body composition, $\beta$-amyloid and DHEAs concentration in elderly women. Korean J. Phys. Educ. 2009, 48, 503-511.

54. Moon, D.S.; Kwon, Y.C. The effect of Taekwondo Exercise on Health-Related Physical Fitness, Cognitive Ability and Dementiainduced Factors in Frail Elderly Women. Korean J. Sports Sci. 2010, 19, 875-887.

55. Shin, J.D. Effects of Difference Exercise Type on Taekwondo Poomsae Training and Walking exercise on Body Compositon, Serum Cholesterol and Dementia Risk Factors in Elderly Obese Women. Korean J. Sports Sci. 2010, 19, 1069-1080.

56. Cho, W.J. The Effects of Taekwondo Poomsae Training on Body Composition, hs-CRP, TNF- $\alpha$ and Adiponectin in Old obese Women. Korean J. Sport 2012, 10, 567-578.

57. Lim, Y.R.; Lee, G.H. The Influence of 12-week Taekwondo Aerobics on Healthy Physical Fitness and Blood Composition in the Middle Ages Woman. Korean J. Sport 2017, 15, 547-555.

58. Zemel, B. Body composition during growth and development. In Human Growth and Development; Elsevier Science: Orando, FL, USA, 2002; pp. 271-294. [CrossRef]

59. Bouchard, C.E.; Shephard, R.J.; Stephens, T.E. Physical Activity, Fitness, and Health: International Proceedings and Consensus Statement. International Consensus Symposium on Physical Activity, Fitness, and Health, 2nd, Toronto, ON, Canada, May 1992; Human Kinetics Publishers: Champaign, IL, USA, 1994. [CrossRef]

60. Nam, S.; Lim, K. Effects of Taekwondo training on physical fitness factors in Korean elementary students: A systematic review and meta-analysis. J. Exerc. Nutr. Biochem. 2019, 23. [CrossRef] [PubMed]

61. Riebe, D.; Ehrman, J.K.; Liguori, G.; Magal, M. American College of Sports Medicine. ACSM's Guidelines for Exercise Testing and Prescription; Wolters Kluwer: Alphen aan den Rijn, The Netherlands, 2018.

62. Lee, S.K.; Kong, M.A.; Kang, H.S. Measurement of exercise intensity during basic movement, kicking and Poomsae of Taekwondo. Korean Soc. Exerc. Physiol. 2003, 12, 727-734. 
63. Ainsworth, B.E.; Haskell, W.L.; Herrmann, S.D.; Meckes, N.; Bassett, D.R.; Tudor-Locke, C.; Greer, J.L.; Vezina, J.; Whitt-Glover, M.C.; Leon, A.S. 2011 Compendium of Physical Activities: A second update of codes and MET values. Med. Sci. Sports Exerc. 2011, 43, 1575-1581. [CrossRef]

64. Alkahtani, S. Comparing fat oxidation in an exercise test with moderate-intensity interval training. J. Sports Sci. Med. 2014, 13, 51. [PubMed]

65. Helgerud, J.; Høydal, K.; Wang, E.; Karlsen, T.; Berg, P.; Bjerkaas, M.; Simonsen, T.; Helgesen, C.; Hjorth, N.; Bach, R. Aerobic high-intensity intervals improve VO2max more than moderate training. Med. Sci. Sports Exerc. 2007, 39, 665-671. [CrossRef] 